\title{
Solubility Determination from Clear Points upon
}

\section{Solvent Addition}

Marloes A. Reus ${ }^{* 广}$, Antoine E.D.M. van der Heijden ${ }^{\dagger+}$, Joop H. ter Horst ${ }^{\S}$

${ }^{\dagger}$ Delft University of Technology, Process \& Energy Department, Intensified Reaction \& Separation Systems, Leeghwaterstraat 39, 2628 CB, Delft, The Netherlands. (*email:

M.A.Reus@tudelft.nl)

$\ddagger$ TNO Defence, Security and Safety, Dept. Energetic Materials, P.O. Box 45, 2280 AA, Rijswijk, The Netherlands

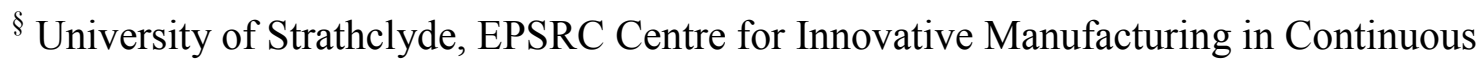

Manufacturing and Crystallisation (CMAC), Strathclyde Institute of Pharmacy and Biomedical

Sciences (SIPBS), Technology and Innovation Centre, 99 George Street, Glasgow G1 1RD, U.K. 


\section{TOC GRAPHIC:}

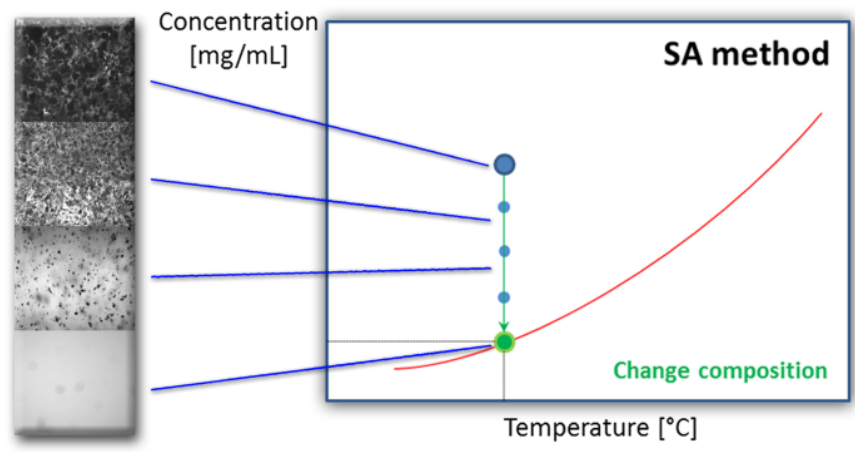


KEYWORDS: Phase diagram, Solubility, Solubility measurement, Saturation concentration, Solvent mixtures.

ABSTRACT: A method is described for determining the solubility of multicomponent crystalline compounds from clear points upon sample dilution at a constant temperature. Clear points are established by continuously adding solvent mixture to a suspension of known composition until a clear solution appears. For validation, this solvent addition method is compared to the traditional equilibrium concentration method at constant temperature and the more recent temperature variation method with which clear point temperatures are determined upon increasing the sample temperature. Solubility data of binary systems ( 1 solute, 1 solvent) measured using the solvent addition method are obtained relatively quickly compared to the equilibrium concentration method. These solubility data are consistent with those of the temperature variation and the equilibrium concentration method. For the temperature variation method the results are dependent on the heating rate. Likewise, for the solvent addition method they are dependent on the addition rate. Additionally, for ternary systems involving anti-solvent or co-crystals, solubilities are determined at a constant temperature using the solvent addition method. The use of the solvent addition method is especially valuable in the case of solvent mixtures and other complex multi-component systems, in which the temperature variation method cannot be applied easily.

\section{Introduction}

In production often a crystallization step is required for purification and final crystalline particulate product formation. ${ }^{1,2}$ The solubility or phase diagram of such compounds is essential information for efficient and reliable crystallization process design and operation. ${ }^{3-6}$ The phase 
diagram indicates the most stable phases at specific compositional and temperature conditions ${ }^{1,4-}$

${ }^{6}$, determines the achievable yield ${ }^{7}$ and enables the monitoring of the supersaturation during the crystallization process $^{7,8}$.

Traditionally the solubility is measured through equilibration of a suspension ${ }^{1}$. The solubility is then equal to the concentration in the equilibrated solution, which can be sampled and determined by for example, a gravimetric method or HPLC (figure 1 (left)). Although the Equilibrium Concentration (EqC) method is widely accepted and considered accurate ${ }^{1}$, it is laborious and time-consuming. Currently, commercial equipment from various suppliers is available that streamlines measurements through a temperature variation (TV) method in which clear points are measured. ${ }^{9-11}$ In the TV method the solubility is changed by changing the temperature, until it matches the concentration. A clear point is then the temperature at which, upon increasing the temperature, a suspension turns into a clear solution. Figure 1 (center) shows the principle of a clear point measurement using the TV method. If the heating rate is sufficiently small, the crystal dissolution rate is fast and the clear point can be assumed to be equal to the saturation temperature. ${ }^{10}$ This TV method is much less labor intensive, much faster and allows for the measurement of the metastable zone width or induction time in the same measurement. However, the TV method loses its advantages when solubility data is required at a specific temperature, for example in phase diagrams with multicomponent mixtures in which the solubility is a function of the concentration of more than 1 component, since the TV method relies on a variable temperature. 


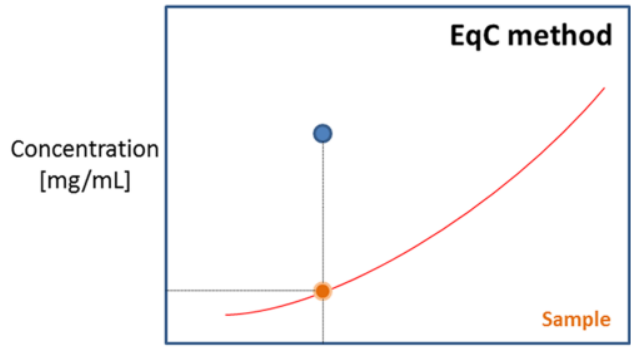

Temperature $\left[{ }^{\circ} \mathrm{C}\right]$

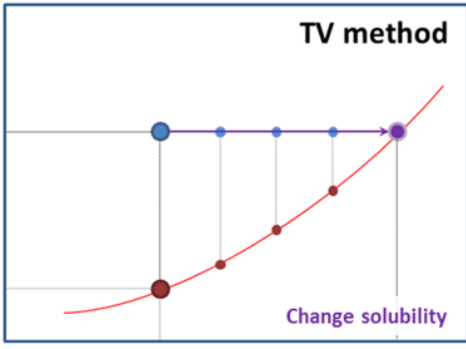

Temperature $\left[{ }^{\circ} \mathrm{C}\right]$

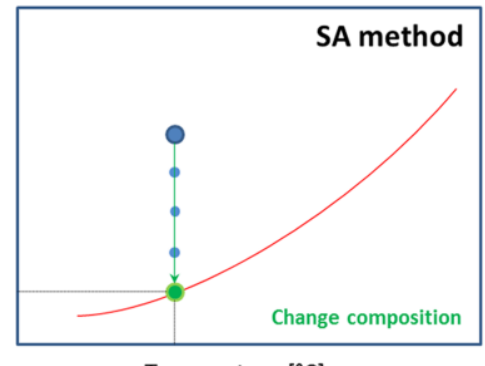

Temperature $\left[{ }^{\circ} \mathrm{C}\right]$

Figure 1. Different principles for solubility measurements using a certain overall composition (Blue dot). Left: EqC method. The equilibrium concentration (orange dot) is determined by analysing the concentration in an equilibrated suspension. Centre: The purple arrow represents the TV method, where the solubility of the system is changed until it corresponds to the overall concentration. Right: The green arrow represents the Solvent Addition (SA) method, where the concentration in the system is continuously decreased until it corresponds to the solubility at constant temperature.

Several groups ${ }^{1,12-14}$ reported another method of determining solubility. Instead of increasing the solubility by increasing the temperature, in this Solvent Addition (SA) method the concentration is decreased by adding solvent (figure 1 (right)). The green arrow in figure 1 represents the SA method. At a constant temperature, starting from a known suspension, solvent is added drop-wise until full dissolution of the material. Also continuous methods have been shown to work, for example by Labarthe et al. ${ }^{15,16}$, predominantly in multicomponent mixtures. However, no account was given as to how parameters like the addition rate must be chosen to obtain reliable data. Additionally, up to now this approach is more labor intensive and time consuming than even the $\mathrm{EqC}$ method and is therefore not widely applied. We intensify this method by making the addition continuous instead of quasi static and by using clear point 
measurements for ease of operation. Additionally, this method can be automated similar to the TV method. We therefore aim to make the method more accessible and more widely applicable.

This method seems particularly useful for determining phase diagrams of complex multicomponent mixtures at constant temperature, since the concentration of each component can be tracked. Here we report the validation of the Solvent Addition method through a study of the solubility of p-Hydroxybenzoic acid (p-HBA) in acetic acid. Additionally, the influence of the solvent addition rate on the accuracy of the SA measurements is determined. Finally the SA method is applied to two multi-component systems; DL-asparagine (DL-Asn) in water-ethanol mixtures, and the carbamazepine - isonicotinamide (CBZ-INA) co-crystal in the solvent ethanol.

\section{Experimental section}

\section{Materials}

p-HBA $(\geq 99 \%)$, INA ( $\geq 99 \%)$, CBZ $(99 \%)$, glacial acetic acid, ethanol (100\%) and DL-Asn ( $\geq 99 \%$ ) were supplied by Sigma Aldrich and used without further purification. Demi water was taken from a Millipore MilliQ system and had a resistivity of approximately $18 \mathrm{M} \Omega \cdot \mathrm{cm}$.

\section{Equilibrium concentration method}

For the EqC method the same protocol was used as reported by Nordström and Rasmuson ${ }^{17}$, where the solution was equilibrated at a constant temperature in a $100 \mathrm{~mL}$ Easymax reactor setup (Mettler Toledo) for 4 days. At various temperatures solution samples of approximately $5 \mathrm{~mL}$ were taken with a syringe, weighed and left to evaporate in a fume hood for at least 14 days. When the crystals appeared dry, they were weighed. Two days later they were weighed again to check for any additional mass loss until the mass was constant.

\section{Temperature variation method}


For the TV method the same protocol was used as by Vellema et al. ${ }^{10}$, using the Crystal16 multiple reactor setup (Avantium Technologies). The samples were agitated using magnetic stirrer bars with a stirring rate of $700 \mathrm{rpm}$. For the p-HBA phase diagram, the temperature was varied between $0-90^{\circ} \mathrm{C}$. For determining the influence of the heating rate on the clear point measurements, concentrations of 136.2 and $161.4 \mathrm{mg} / \mathrm{mL}$ of p-HBA in acetic acid were used in a temperature range of $0-60^{\circ} \mathrm{C}$.

\section{Solvent addition method}

For the solvent addition experiments a Crystalline Multiple Reactor setup (Avantium Technologies) was used to provide a constant temperature and stirring rate (300 rpm), and to monitor the dissolution process by video. The starting volume was $2 \mathrm{~mL}$ of suspension. The solution was allowed to equilibrate for approximately 30 minutes prior to the solvent addition.

A rough estimate of the upper level of the equilibrium concentration in the co-crystal system was made using the pure component solubilities ${ }^{18}$. If a rough educated guess of the equilibrium concentration was not possible, a small amount of crystalline material was taken and small known amounts of solvent were added in steps until the material is dissolved. The amount of solvent added and the amount of crystalline material lead to an estimate of the solubility. Starting points for other compositions or temperatures in the same system were estimated from neighboring measurements, by either increasing or decreasing the concentration compared to the neighboring measurement. For example, for a solubility measurement at $30^{\circ} \mathrm{C}$, a starting composition was used with a concentration of approximately 1.5 times the concentration in the starting composition of the measurement at $20^{\circ} \mathrm{C}$. As a rule of thumb the solubility doubles when the temperature increases by $20^{\circ} \mathrm{C}^{19}$, which was used as a guideline. A relatively dense 
suspension was chosen as a starting point for the SA measurement, at which the amount of solid added to the solvent was 1.3 to 3 times higher than the rough estimate.

Solvent was added using an Aitecs 2016 syringe infusion pump (volumetric accuracy of \pm $2 \%$ ). Clear points were determined by visual analysis of the suspension pictures taken every 30 seconds. This method was preferred over the turbidity measurements in the same device, because of the better detection limit.

For larger scale solvent addition measurements a $100 \mathrm{~mL}$ Easymax reactor (Mettler Toledo) was used with overhead stirring (250 rpm), combined with an SP-50 dosing unit. Clear points in the Easymax were determined by FTIR measurements using the ReactIR system (Mettler Toledo) equipped with an $\mathrm{AgX}$ probe with diamond tip and an $\mathrm{LN}_{2} \mathrm{MCT}$ detector. Peaks at

$1168 \mathrm{~cm}^{-1}$ and at $1612 \mathrm{~cm}^{-1}$ were used to monitor the concentration changes of the compound in solution. The concentration remained constant until all crystalline material was dissolved. The start of a concentration decrease marked the clear point, which was determined by the intersection of the horizontal line (constant concentration) and the sloped line (decreasing concentration).

\section{Characterization}

The crystalline phase was analyzed by X-ray Powder Diffraction (XRPD), using a Bruker AXS D2-Phaser with a Bragg-Brentano focusing geometry and a CuKa1 radiation. Measurements were taken between $5<2 \theta<50^{\circ}$. An optical microscope (Leica Nikon Optiphot 200) was used for the analysis of the crystal shape. 


\section{Results}

p-HBA is a white crystalline solid and is mostly known for its esters, which are used as preservatives. The SA method was used to determine its solubility in acetic acid. In a small volume $\left(V_{\text {start }}=2 \mathrm{~mL}\right)$, the SA method was applied at 3 different temperatures while using a solvent addition rate of $R_{a}=0.5 \mathrm{~mL} / \mathrm{h}$. While adding acetic acid, the suspension was monitored by video images. From figure 2 the decreasing suspension density upon solvent addition and the clear point after addition of $0.70 \mathrm{~mL}$ of acetic acid are evident. More pictures were recorded during the experiment for accurate determination of the clear point, but are left out of figure 2 for clarity. The clear point lies between 0.696 and $0.706 \mathrm{~mL}$ of added acetic acid, resulting in a solubility of $94.5 \pm 0.2 \mathrm{mg} / \mathrm{mL}$.

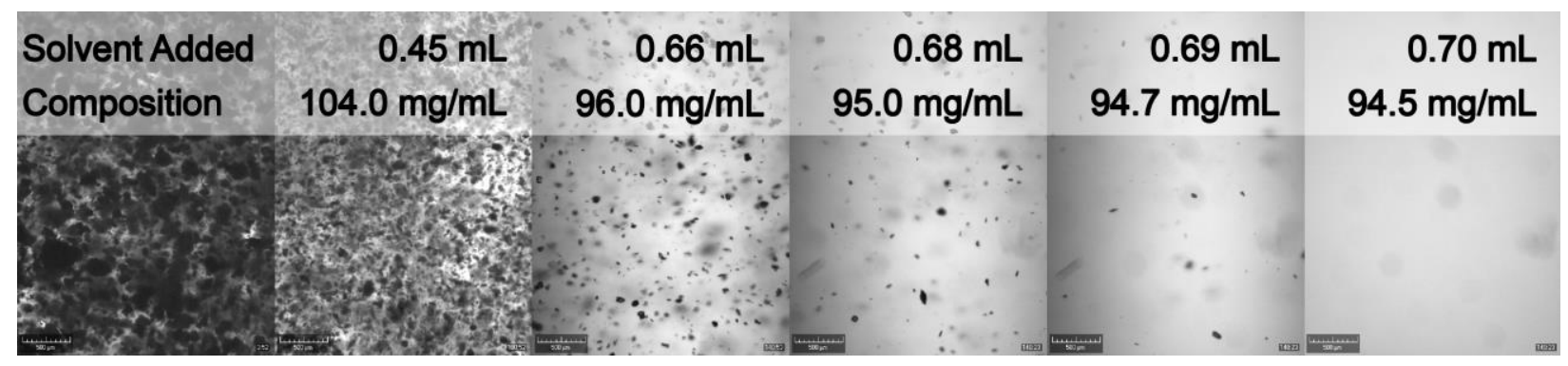

Figure 2. Visualization of the suspension density decrease of $255.2 \mathrm{mg} / \mathrm{mL}$ p-HBA in $2 \mathrm{~mL}$ acetic acid upon acetic acid addition. After addition of $0.70 \mathrm{~mL}$ of acetic acid a clear solution was obtained.

From 2 or 3 measurements per temperature, average clear points were determined at 94.3 , 113.7 and $131.7 \mathrm{mg} / \mathrm{mL}$ at temperatures of 20,30 and $40^{\circ} \mathrm{C}$, respectively. These data are shown in figure 3 as green diamonds. The solubilities were reproducible within $0.4 \mathrm{mg} / \mathrm{mL}$. 


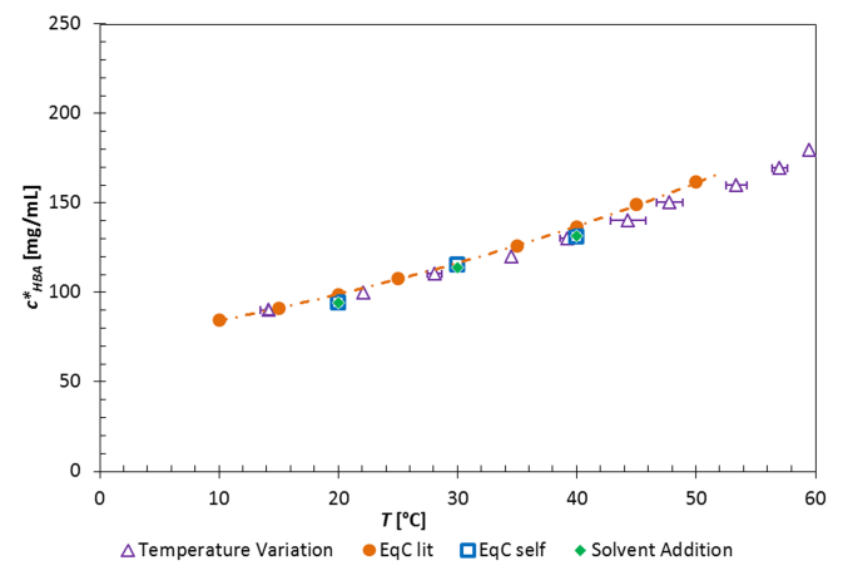

Figure 3. Phase diagram of $\mathrm{p}-\mathrm{HBA}$ in acetic acid measured by the $\mathrm{EqC}$ method $(\bullet=$ literature $\operatorname{data}^{17}, \square=$ own EqC measurements), TV method $(\Delta)$ and SA method $(\diamond)$. For the TV and SA methods, heating and addition rates of $0.3^{\circ} \mathrm{C} / \mathrm{min}$ and $0.3 \mathrm{~mL} / \mathrm{h}$ were used, respectively.

\section{Comparison with other solubility measurement methods}

For p-HBA in acetic acid, the phase diagram was also determined using the TV method $(\Delta)$ and the EqC method ( $\square$ ). Both datasets are in good agreement with each other. The standard deviation in the EqC data is very small, which is expected due to the number of samples taken (15 at each temperature) and the long time allowed for equilibration. In the TV data the standard deviation is somewhat larger, especially at higher temperatures. This larger deviation may be due to the dynamic nature of the measurements and to the number of samples that make up the average (3 clear points were determined per concentration). The SA measurements $(\diamond)$ are in good agreement with the data obtained by the EqC and TV methods. Additionally, previously measured data by Nordström and Rasmuson ${ }^{17}$, using the EqC method are plotted in figure $3(\bullet)$. Our measurements fit well with these data. 

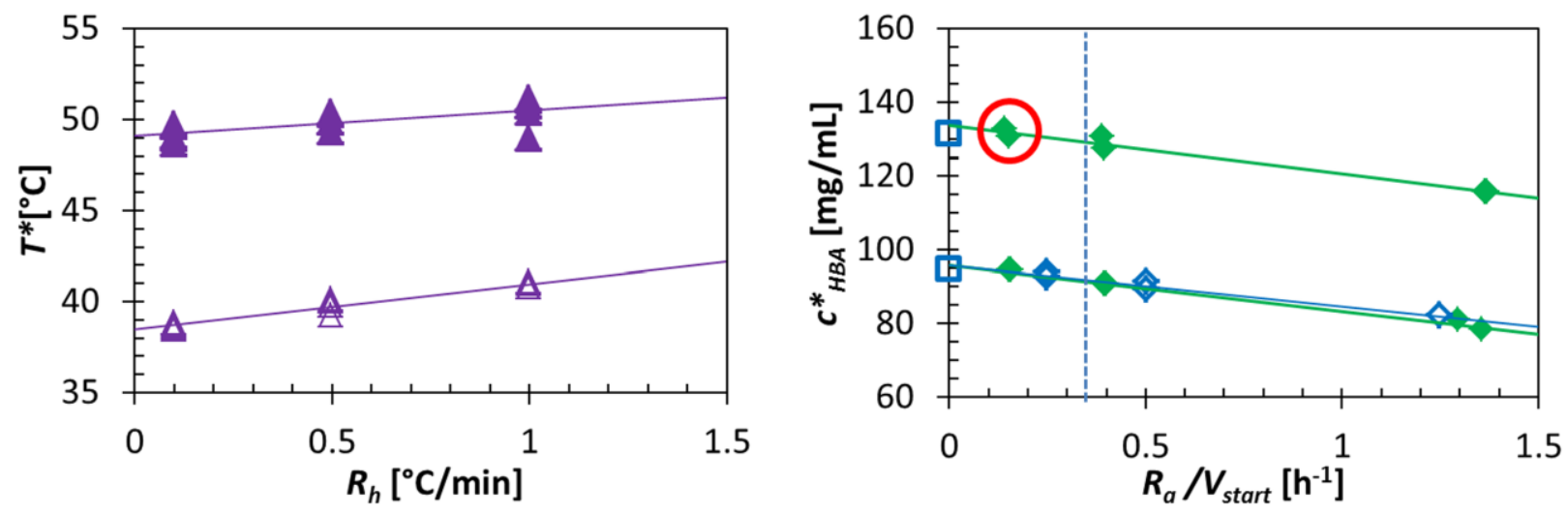

Figure 4. Left: Influence of heating rate $\left(R_{\mathrm{h}}\right)$ on clear point temperatures $\left(T^{*}\right)$ of $\mathrm{p}-\mathrm{HBA}$ in acetic acid during TV measurements using 2 different concentrations $\left(c_{\mathrm{HBA}}=136.2 \mathrm{mg} / \mathrm{mL}(\Delta)\right.$ and $161.4 \mathrm{mg} / \mathrm{mL}(\Delta))$. Right: Influence of addition rate $\left(R_{\mathrm{a}}\right)$ on clear point concentration of $\mathrm{p}$-HBA $\left(c^{*}{ }_{\mathrm{HBA}}\right)$ in acetic acid during SA measurements at 2 different temperatures $\left(T=20^{\circ} \mathrm{C}\right.$ and $\left.40^{\circ} \mathrm{C}\right)$ and at small $\left(V_{\text {start }}=2 \mathrm{~mL}\right)(\diamond)$ and large scale $\left(V_{\text {start }}=40 \mathrm{~mL}\right)(\diamond)$. In the right graph our EqC measurements ( $\square$ ) are provided for comparison. For the measurements in the circle an addition rate of $0.3 \mathrm{~mL} / \mathrm{h}$ was used, the dashed vertical line represents the addition rate of $R_{a}=0.75 \mathrm{~mL} / \mathrm{h}$ for a starting volume of $2 \mathrm{~mL}$.

Heating rate in the TV method. For any solubility measurement using the dynamic techniques (TV and SA), it is mainly important that the rates of change are small enough for the dissolution to keep up with the change. Therefore, with both methods a sensitivity analysis is performed for the p-HBA in acetic acid system, where the rates of change are plotted versus the recorded clear points.

Clear point temperatures were measured for p-HBA in acetic acid using the TV method. Figure 4 (1) shows the measured clear point as a function of the heating rate $\left(R_{h}\right)$. For a concentration of $c_{H B A}=136.2 \mathrm{mg} / \mathrm{mL}$ six clear point temperatures were measured, averaging at $T^{c}=39.9^{\circ} \mathrm{C}$ with a standard deviation of $0.3^{\circ} \mathrm{C}$. It is observed that higher heating rates yield higher clear point 
temperatures, thus lower apparent solubilities. This is mainly related to the interplay between the overall crystal dissolution rate and the applied heating rate, because the rate of change in the solubility depends on the heating rate. If the heating rate is too high, the dissolution of the crystals is not fast enough to keep up with the increasing solubility, the crystals will ultimately dissolve at a higher temperature. In those cases, a clear point temperature will be measured that is higher than the actual saturation temperature.

Upon decreasing $R_{h}$, the clear point would approach the saturation temperature, as seen in figure 4 (l). If the heating rate is sufficiently low the crystals have sufficient time to dissolve and the solution approaches thermodynamic equilibrium at each temperature. Ideally a heating rate close to $R_{h}=0^{\circ} \mathrm{C} / \mathrm{min}$ would lead to clear point temperatures that are equal to the saturation temperature. A linear extrapolation for the sample with an overall concentration of $136.2 \mathrm{mg} / \mathrm{mL}$ leads to a saturation temperature of $T^{*}=38.5 \pm 0.4^{\circ} \mathrm{C}$ (figure 4 (1)). Using a heating rate of $R_{h}=0.5^{\circ} \mathrm{C} / \mathrm{min}$ the measured clear point is $T^{c}=39.9^{\circ} \mathrm{C} \pm 0.3^{\circ} \mathrm{C}, 1.4^{\circ} \mathrm{C}$ higher than the extrapolated saturation temperature. For a sample with an overall concentration of $161.4 \mathrm{mg} / \mathrm{mL}$ a saturation temperature of $T^{*}=49.1 \pm 0.7^{\circ} \mathrm{C}$ is extrapolated. Using a $R_{h}$ of $0.5^{\circ} \mathrm{C} / \mathrm{min}$ the measured clear point is $T^{c}=49.9 \pm 0.4^{\circ} \mathrm{C}, 0.8^{\circ} \mathrm{C}$ higher than the extrapolated saturation temperature. Based on both measurements, we assumed a $R_{h}=0.3^{\circ} \mathrm{C} / \mathrm{min}$ to provide us with clear point temperatures that fall within $1.0^{\circ} \mathrm{C}$ of the actual saturation temperature $\left(T^{c}=49.7\right.$ and $39.5^{\circ} \mathrm{C}$ for the high and low concentrations, respectively). A typical single clear point measurement using this heating rate would take around 1 to 4 hours. Measuring multiple vials simultaneously and including duplo measurements of the same recrystallized samples enables the construction of a solubility line within one day. 
Addition rate in the SA method. Using the SA method clear point concentrations of the same system (p-HBA in acetic acid) were measured. The SA method was applied at 2 different temperatures while using solvent addition rates varying from 0.3 to $5.5 \mathrm{~mL} / \mathrm{h}$. Figure 4 (r) shows the relation between the addition rate $\left(R_{\mathrm{a}}\right)$ and the measured clear point concentrations. The clear point concentrations are plotted against the addition rate $\left(R_{\mathrm{a}}\right)$ divided by the initial volume $\left(V_{\text {start }}\right)$ to enable the comparison of measurements at different scales. It shows that lower addition rates lead to higher clear point concentrations. Analogous to the trend in the clear point temperature variation with the heating rate, the clear point concentration is determined by a balance between the dissolution rate and the concentration dilution rate, the latter of which depends on the $R_{a}$. In the solvent addition method the Addition Rate $\left(R_{\mathrm{a}}\right)$ is to some extent the equivalent of the heating rate in the TV method. If the $R_{a}$ is too high, the dissolution of the crystals is not fast enough to keep up with the decreasing concentration.

A linear extrapolation for the samples at $T=20$ and $40^{\circ} \mathrm{C}$ lead to a saturation concentration of $c^{*}{ }_{\mathrm{HBA}}=95.8 \pm 0.6 \mathrm{mg} / \mathrm{mL}$ and $133.8 \pm 0.9 \mathrm{mg} / \mathrm{mL}$ (figure $4(\mathrm{r})$ ). For the equipment, chemicals and $2 \mathrm{~mL}$ starting volume, clear point data within $5 \%$ of the saturation concentration are obtained at addition rates around $R_{a}=0.75 \mathrm{~mL} / \mathrm{h}$ or lower. A typical single clear point measurement using solvent addition, starting with $2 \mathrm{~mL}$ to which $4 \mathrm{~mL}$ is added, would then take around 2 to 3 hours.

In principle the initial volume should not affect the clear point measured if the appropriate addition rate is used. To test this, SA measurements were performed at $T=20^{\circ} \mathrm{C}$ at a larger scale, in which the starting volume was $40 \mathrm{~mL}$. The corresponding addition rate would then be $15 \mathrm{~mL} / \mathrm{h}$. The clear point was determined using FTIR measurements. The peaks at $1168 \mathrm{~cm}^{-1}$ and at $1612 \mathrm{~cm}^{-1}$ were used to monitor the concentration changes of the compound in solution. An 
example of such a measurement is shown in figure 5. Upon addition of acetic acid, in this case with an addition rate of $R_{a}=10 \mathrm{~mL} / \mathrm{h}$, the concentration of $\mathrm{p}-\mathrm{HBA}$ in solution remained constant (saturated), because the dilution was compensated with the dissolution of crystals. After a certain amount was added, the concentration decreased, because all crystals in the suspension were dissolved, which marked the clear point. The precise clear point was determined by the intersection of the slopes. The clear point for this particular measurement was found after addition of $12.5 \mathrm{~mL}$ of acetic acid. The concentration of $\mathrm{p}-\mathrm{HBA}$ in the crystallizer is plotted in the same figure and found on the second y-axis as $c_{H B A} *=0.94 \mathrm{~g} / \mathrm{mL}$.

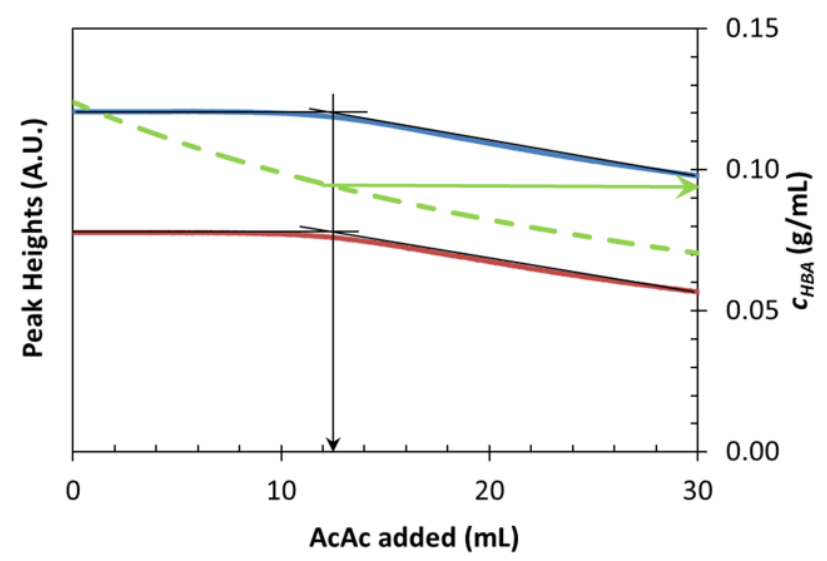

Figure 5. Trends of IR spectrum peak heights at $1168 \mathrm{~cm}^{-1}$ (blue) and $1612 \mathrm{~cm}^{-1}$ (red) taken during a SA experiment with p-HBA in acetic acid at $20^{\circ} \mathrm{C}$ with an acetic acid addition rate of $10 \mathrm{~mL} / \mathrm{h}$ over a starting volume of $40 \mathrm{~mL}$. The green dashed data represents the concentration of p-HBA in the crystallizer. The clear point in this experiment was determined as $c_{H B A} *=0.940 \mathrm{~g} / \mathrm{mL} \pm 0.005 \mathrm{~g} / \mathrm{mL}$, after addition of $12.5 \mathrm{~mL} \pm 0.3 \mathrm{~mL}$ of acetic acid.

These results, alongside the small scale tests, are shown in figure $4(\mathrm{r})$ and are in good agreement with those of the small scale tests, yielding a clear point at $R_{a} / V_{\text {start }}=0 \mathrm{~h}^{-1}$ of $95.9 \pm$ $0.6 \mathrm{mg} / \mathrm{mL}$. These measurements confirm that similar results are obtained at different scales 
using different detection techniques with the SA method as long as the same $R_{a} / V_{\text {start }}$ is used. The method therefore can be used at any scale, as long as the addition rate is chosen accordingly.

Comparing heating and addition rate. In order to allow the comparison between the TV and SA data in figure 3, the rates of change used in both methods should be equivalent at the clear point. For both methods the rate of change can be expressed as the relative dilution rate, $d\left(c_{i} / c_{i}{ }^{*}\right) / d t$ and is assessable at the clear point, i.e. where $\left(c_{i} / c_{i}{ }^{*}\right)=1$.

In TV measurements, by changing the temperature, the solubility of the compound $\left(c_{i}^{*}\right)$ is increased in order to completely dissolve the crystals while the overall composition $\left(c_{i}\right)$ remains unchanged. The solubility can be described as an exponential function of the temperature:

$$
c_{i}^{*}(T)=a \exp (-b T)
$$

When the composition in the crystallizer does not change, the supersaturation ratio can then be expressed as:

$$
\frac{c_{i}}{c_{i}^{*}(T)}=\frac{c_{i}}{a} \exp (-b T)
$$

In eq. $1, a$ and $b$ are fitting parameters. The temperature $T(t)$ in degrees Celsius is changed over time according to:

$$
T=T_{0}+R_{h} t
$$

Combining equations 1 and 2 and taking the derivative over time yields the rate of change for TV measurements as a function of the heating rate $R_{\mathrm{h}}$ :

$$
\frac{d\left(c_{i} / c_{i}^{*}(T)\right)}{d t}=-b R_{h}\left(c_{i} / c_{i}^{*}(T)\right)
$$

Which shows that the relative dilution rate at $\mathrm{c} / \mathrm{c}^{*}=1$ is a linear function of the heating rate. 
From our experimental data, the fitting parameters were $a=70.5 \mathrm{mg} / \mathrm{mL}$ and $b=0.0157 /{ }^{\circ} \mathrm{C}$. Starting at $T_{0}=20^{\circ} \mathrm{C}$ with a concentration $c_{i}=131 \mathrm{mg} / \mathrm{mL}$, the clear point should be reached at $40^{\circ} \mathrm{C}$. Using a heating rate of $R_{h}=0.30^{\circ} \mathrm{C} / \mathrm{min}$ gives a relative dilution rate of $d\left(c_{i} / c_{i}^{*}\right) / d t=-4.7 \cdot 10^{-3} / \mathrm{min}$

In the performed SA measurements, by addition of solvent, the overall concentration of the compound in the vessel $\left(c_{i}\right)$ is decreased in order to dissolve the crystals while the solubility remains unchanged. In case of complex multicomponent mixtures where solvent mixtures are added, however, the solubility may change. For the performed SA measurements the supersaturation ratio is expressed via the change in concentration $c_{\mathrm{i}}(t)$ over time due to the changing volume $V(t)$ of solvent:

$$
\frac{c_{i}}{c_{i}^{*}}=\frac{c_{i, 0}}{c_{i}^{*}} \cdot \frac{V_{0}}{V(t)}(5)
$$

in which $c_{i, 0}$ is the initial concentration of compound in the vial and $V_{0}$ is the initial volume of solvent. The volume of solvent in the vial is expressed as:

$$
V(t)=V_{0}+R_{a} t
$$

Substituting eq. 5 into 4 and taking the derivative over time yields the rate of change for SA measurements:

$$
\frac{d\left(c_{i} / c_{i}^{*}\right)}{d t}=-\frac{\left(\frac{c_{i, 0}}{c_{i}^{*}}\right) V_{0} R_{a}}{\left(V_{0}+R_{a} t\right)^{2}}
$$

Eq. 6 shows that at a constant addition rate, the relative dilution rate decreases with increasing sample volume $V$. At a temperature of $40^{\circ} \mathrm{C}$, the solubility is $c_{i}{ }^{*}=131 \mathrm{mg} / \mathrm{mL}$. With an initial volume of $V_{0}=2 \mathrm{~mL}$, an initial overall concentration of $c_{0}=163 \mathrm{mg} / \mathrm{mL}$, and an addition rate of $R_{\mathrm{a}}=0.3 \mathrm{~mL} / \mathrm{h}(0.005 \mathrm{~mL} / \mathrm{min}$, experiments in red circle in figure $4(\mathrm{r}))$ a relative dilution rate 
of $-2.0 \cdot 10^{-3} / \mathrm{min}$ is calculated at the clear point. This falls in the same order of magnitude as the rate of change in solubility for the TV measurements, which means that the data can fairly be compared. Since the rate of change in the SA measurements is smaller than in the TV measurements, it is not surprising that the accuracy of the SA data is higher. In this system at $40^{\circ} \mathrm{C}$, a similar change in supersaturation ratio can be obtained to that of the TV measurement if an addition rate of $R_{a}=0.75 \mathrm{~mL} / \mathrm{h}$ (dashed blue line in figure $4(\mathrm{r})$ ) is used. The rate of change in supersaturation ratio in that case is $d\left(c_{i} / c_{i}{ }^{*}\right) / d t=-5.0 \cdot 10^{-3} / \mathrm{min}$ at the clear point concentration.

\section{The SA method in more complex mixtures}

The TV method is the fastest method for obtaining solubility data of pure components in single solvents. However, when multicomponent mixtures are investigated and a phase diagram at a single, fixed temperature is required, the TV method loses its advantages over the SA method. We measured the solubility of crystalline compounds in multicomponent mixtures containing multiple solvents (anti-solvent crystallization) and multiple solutes (co-crystallization).

Phase diagram for anti-solvent crystallization. Asparagine (Asn) is one of the most common amino acids and appears as a white crystalline solid. It dissolves well in water, but not in alcohols. Therefore, anti-solvent crystallization can be performed with this compound. The phase diagram at $T=20^{\circ} \mathrm{C}$ of DL-Asn in water with ethanol as anti-solvent is determined by the SA method and presented in figure 6, where the solubility of DL-Asn in the solvent mixture (green data) is plotted against the solute excluded mass fraction of ethanol, $x_{E}$. 


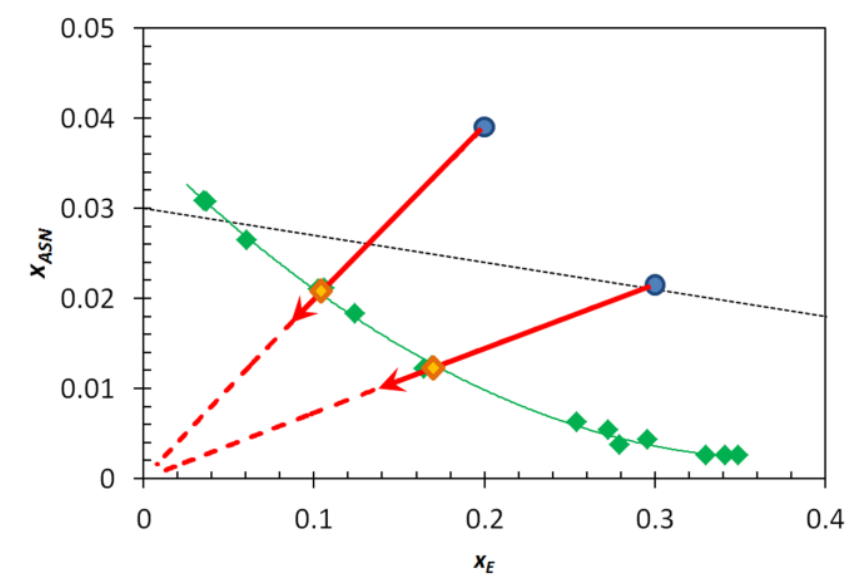

Figure 6. Solubility of anhydrous DL-Asn in the solvent - anti-solvent system water - ethanol. The measured mass fraction $x_{A S N}$ of DL-Asn at $20^{\circ} \mathrm{C}$ is plotted versus the mass fraction $x_{\mathrm{E}}$ of ethanol on solute free basis. The red arrows indicate the overall composition during the measurement of the clear points (orange diamonds) starting at an overall composition in the blue points. The black dashed line represents the addition of pure ethanol to a solution of DL-Asn in water during an anti-solvent crystallization experiment.

The red arrow in figure 6 demonstrates the measurement method. For the solubility of Asn in the solvent - anti-solvent system water - ethanol, the starting point was chosen such that the mass fraction of Asn in an ethanol-water mixture of low ethanol concentration was approximately equal to the solubility in pure water. The mass fraction of Asn corresponding to the solubility measured in this measurement was used as a starting point for a measurement with a higher ethanol starting concentration. If necessary, extra points were added in between compositions of previous measurements. Starting at an overall suspension composition corresponding to the blue point, pure water was added using an addition rate $R_{a, V}=1 \mathrm{~mL} / \mathrm{h}$ to a 2 $\mathrm{mL}$ starting volume. This addition rate is slightly larger than the addition rate applied for the p-HBA - acetic acid system in figure 3. However, it was found experimentally, using the method displayed in figure 4 that $1 \mathrm{~mL} / \mathrm{h}$ was a sufficiently low addition rate. The overall composition 
then follows the red line towards the origin of the graph. The orange diamond at the point $(0.12$; 18.6) corresponds to the clear point. The larger spread in data points around an ethanol mass fraction of 0.3 is most likely because in some points the starting suspension was chosen rather close to the solubility line, which slightly decreases the accuracy since interval between the suspension pictures was not decreased.

Additionally, an example is given of the dilution path during an anti-solvent crystallization process that starts with an initial solution with a DL-Asn mass fraction of 0.30 in water, to which pure ethanol is added (black dashed line in figure 6). This phase diagram allows for the tracking of supersaturation during anti-solvent crystallization processes and is a valuable tool in determining the optimal amount of ethanol to add to a starting solution in order to achieve a high yield while keeping the added anti-solvent at a minimum. Since DL-Asn forms a monohydrate in water and crystallizes in anhydrous form from ethanol, the crystals in suspensions at $20^{\circ} \mathrm{C}$ were analysed by XRPD. Already at ethanol fractions as low as $x_{E}=0.03$ the anhydrous form was the predominant crystal form.

Co-crystal Phase Diagram. Carbamazepine (CBZ) is a drug that suffers from limited bioavailability due to its dissolution behaviour. One of the solutions for this problem is the formation of co-crystals ${ }^{20}$. Isonicotinamide (INA) is a suitable conformer for $\mathrm{CBZ}^{21}$. For the reliable crystallization of co-crystals it is important that the composition in the crystallizer lies in the co-crystal region of the phase diagram ${ }^{18}$. A simplified version of the phase diagram of this CBZ-INA co-crystal system in ethanol at $T=25^{\circ} \mathrm{C}$ was determined by ter Horst and Cains ${ }^{21}$. This phase diagram was constructed by fitting the data from TV measurements at different temperatures ( $\boldsymbol{\Delta}$ in figure 7 ) with the Van 't Hoff equation for co-crystals:

$$
\ln \left(x_{a} x_{b}\right)=-\frac{\Delta H}{R}\left(\frac{1}{T}-\frac{1}{T_{0}}\right)
$$


Extrapolation of the data to $T=20^{\circ} \mathrm{C}$ leads to a solubility product $\left(x_{a} x_{b}\right)=37.1 \cdot 10^{-6}$ (the point $\Delta$ in figure 7), which in the ideal case is constant at constant temperature (purple dashed curve). In this method it is assumed that the solutes do not influence each other's pure solubilities in the pure component regions. This means that straight lines, perpendicular to each axis represent the pure component solubilities of INA and CBZ. The pure component solubilities at $T=20^{\circ} \mathrm{C}$ are the result of the extrapolation of the pure component solubility lines determined by the TV method. However, ter Horst et al. ${ }^{18}$ also found that the system does not show ideal behaviour in the pure component regions, i.e. the solutes do influence each other's solubility in the pure component region of the phase diagram.

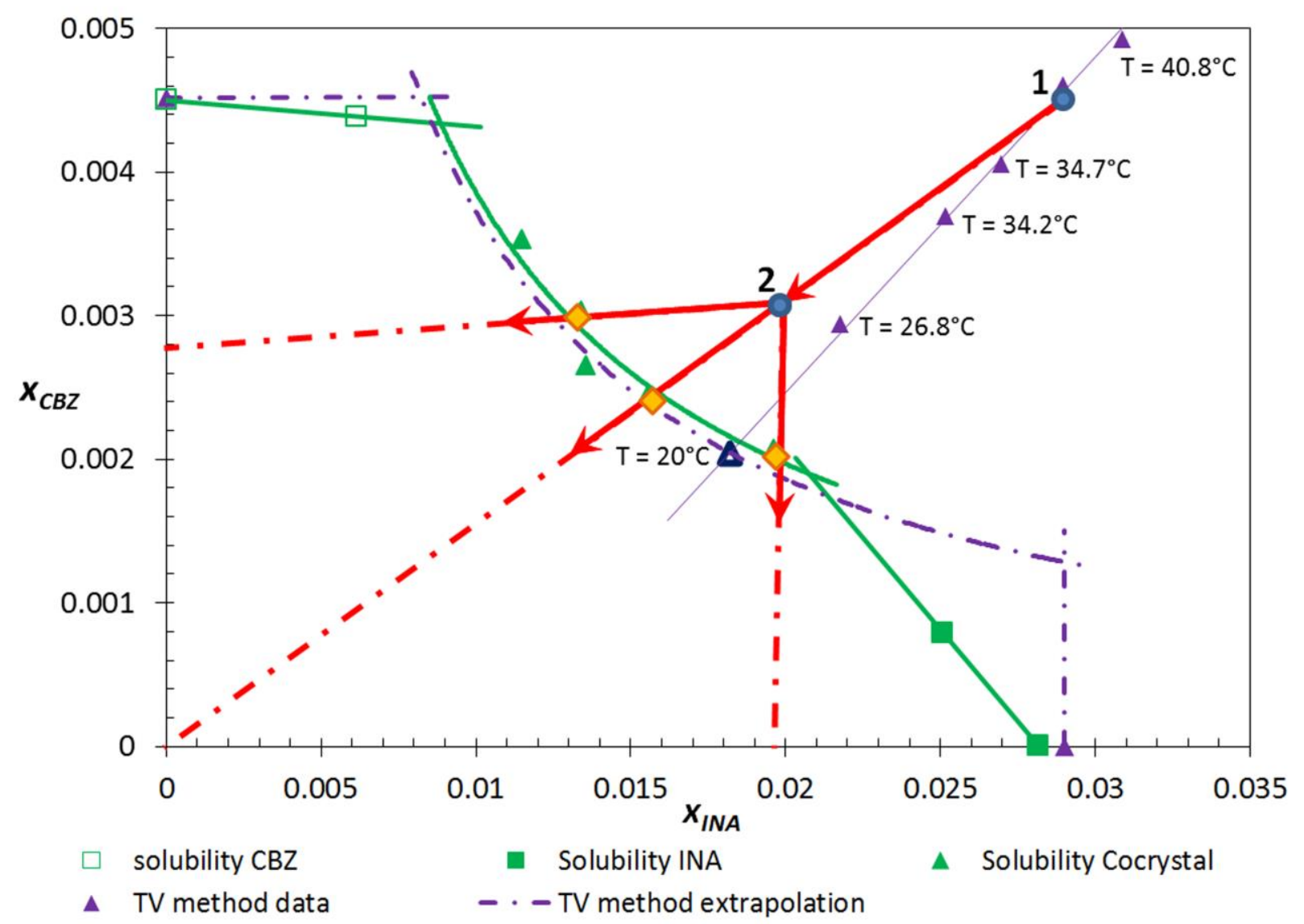

Figure 7. Phase diagram of the INA-CBZ co-crystal in ethanol. The purple triangles are TV data, the purple dotted lines represent the phase diagram constructed at $T=20^{\circ} \mathrm{C}$ based on the 
extrapolation from the TV measurements. The green markers represent SA measurements at $T=20^{\circ} \mathrm{C}$. The blue dots are starting points in the SA methodology, the red arrows depict examples of the dilution path in a number of clear point measurements (orange diamonds) measured by the SA method.

The co-crystal phase diagram was also determined using the SA method. The red arrows in the figure show the measuring protocol used for obtaining the data in the co-crystal region. The method starts by creating a suspension of the co-crystals in solvent by making a starting sample composition of both compounds at their pure component solubility (at $20^{\circ} \mathrm{C} x_{\mathrm{INA}} *=0.028$ and $x_{\mathrm{CBZ}}{ }^{*}=0.0045$, blue point 1$)$ in $2 \mathrm{~mL}$ solvent. The pure crystalline forms dissolved, while cocrystallization occurred from this solution, since this composition is located in the co-crystal region and is supersaturated there ${ }^{18}$. With this suspension, solvent addition using pure solvent was started with an addition rate of $0.5 \mathrm{~mL} / \mathrm{h}$. However, the suspension was rather dense which resulted in a required added volume for complete dissolution that exceeded the volume of the crystallizer. A new starting mixture of $2 \mathrm{~mL}$ was created (blue point 2) having the same composition as the final composition of the previous experiment. This point 2 was the starting point for other clear point measurements, by adding not pure solvent but an undersaturated solution of one of the pure compounds. In this way, such phase diagrams can be constructed without prior knowledge about their shape.

The green markers in figure 7 represent the measurements with the SA method at $T=20^{\circ} \mathrm{C}$. The co-crystal solubility as well as the pure component solubilities are determined using this method. The data points in the co-crystal region have approximately the same solubility products $\left(\left(x_{a} x_{b}\right)=39 \cdot 10^{-6} \pm 10^{-6}\right)$. The solubility products of the data points in the pure component regions deviate significantly from this value. 
In figure 7, it can be seen that the TV data and the SA data agree well in the co-crystal region. The results from the methods do deviate significantly in the pure component regions. While the solubility of CBZ is only slightly affected by the presence of INA in the solution, the solubility of INA decreases significantly in the presence of relatively small concentrations of CBZ. To account for these influences with the TV method many measurements are required. ${ }^{10}$ The SA method is thus suitable for investigating non-ideal behaviour in multi-component systems.

\section{Discussion}

Next to the already accepted methods for determining solubility lines, our SA method proves to be a potential technique. Although our SA method needs as many experiments as the $\mathrm{EqC}$ method and is therefore not as efficient as the TV method, the measurements are less laborious and time consuming than the $\mathrm{EqC}$ method, with the additional advantage that they can be automated. A crucial point for both the TV and the SA method is the determination of the clear point. In this paper the determination of the clear points was done by turbidity measurements, video images and FTIR concentration measurements for $1 \mathrm{~mL}, 6 \mathrm{~mL}$ and $100 \mathrm{~mL}$ samples, respectively. The detection limit for each technique is different and can therefore significantly influence the accuracy of the measurements. For example, turbidity measurements are less accurate at larger sample volumes, making other detection techniques preferable.

One of the challenges in using the SA method, similar to the TV method, is determining the starting composition in the measurement. For systems where no literature data is available, the first starting point must be determined by making a fast estimate of the solubility using a SA measurement with high $R_{a}$, as described in the experimental section. This initial estimate takes up to 30 minutes, including the equilibration time of the suspension prior to the measurement. 
The strongest point of the SA method lies in its application in (complex) multi-component mixtures, in which the composition at any 2-phase to 1-phase transition point at a constant temperature can be determined. Additionally, if the solubility is slightly impacted by temperature changes, for instance in salt or protein systems, the SA method can be preferred as well.

Figure 8 displays the schematic quaternary phase diagram of a hypothetic system containing 2 solutes (A and B) and two solvents (S and AS), in which A and B can co-crystallize and AS is an anti-solvent for all solid phases. The pyramid shows the pure components A, B, S and AS at each corner point. The green areas in figure 8 mark the co-crystal region, the lilac areas mark the regions where the pure components A or B crystallize, the blue areas mark a clear solution and the white areas mark the regions where the co-crystal is present together with either A or B. In the upper left part of figure 8 the sides of the pyramid are unfolded to show each ternary phase diagram out of which the quaternary phase diagram is built up. This is a hypothetic example of a complex multi-component system where SA is ideally suited to provide information about both the solubility of the solid phases A, B and AB in the solvent mixture (e.g. in a system where A and $\mathrm{B}$ are enantiomers). The SA method can be used to determine the phase transitions marked red inside the pyramid. An example of a proposed measurement is given in figure 8 from the blue dot to pure S. Additionally, a projection of this measurement is plotted as a red dashed arrow on each face of the fold out. The blue dot is an example of where the solvent addition measurement can be started. The red arrow depicts the solvent addition pathway, where pure solvent is added to the suspension in the co-crystal region but other compositions can be added as well. The orange diamond represents the clear point. The dotted line on the base of the pyramid is a projection of the dilution pathway and meant as a guideline. 
The SA method, similar to the TV method, results in an equilibrium composition at a certain temperature. However, in the case of polymorphs, co-crystals or other systems, it is unclear which equilibrium is measured without the use of an additional analysis tool. Especially in the neighborhood of a triphasic domain, the equilibrium composition related to a metastable rather than the stable phase could be determined, for instance due to a smaller dissolution rate of the metastable phase. In the case of Asn, there is a region in the phase diagram where the hydrate is the stable form and a region where the hydrate and anhydrous form coexist. Care was taken that only the solubility of the anhydrous Asn was measured. This was confirmed by taking XRPD measurements of crystals crystallized under conditions similar (same temperature, same solvent composition, slightly higher solid content) to those during the SA measurements .

Transitions from triphasic to biphasic domains and reversibly can only be followed with the SA method if a detection technique is used that enables identification between the different solid phases. In a triphasic domain with 2 crystalline phases the composition in solution would be in the polysaturated solution point. Addition of solvent would only change the composition in solution when one of the crystalline phases has disappeared (when going from triphasic to biphasic domains). This change can be detected by spectroscopic techniques. IR and Raman spectroscopy can also be used to determine the contents of the solid phases present until dissolution, which would be beneficial if the solid form exhibits polymorphism. In such case the dissolving polymorph can be identified. In systems where the SMT process is fast ${ }^{2,}{ }^{22}$, the transformation can be followed prior to dissolution. In slower systems ${ }^{23}$, the solubility of the metastable polymorph could be measured. Especially when the disappearing phase can be identified, the SA method is an important addition to the currently used methods for determining phase diagrams of complex multicomponent systems. 


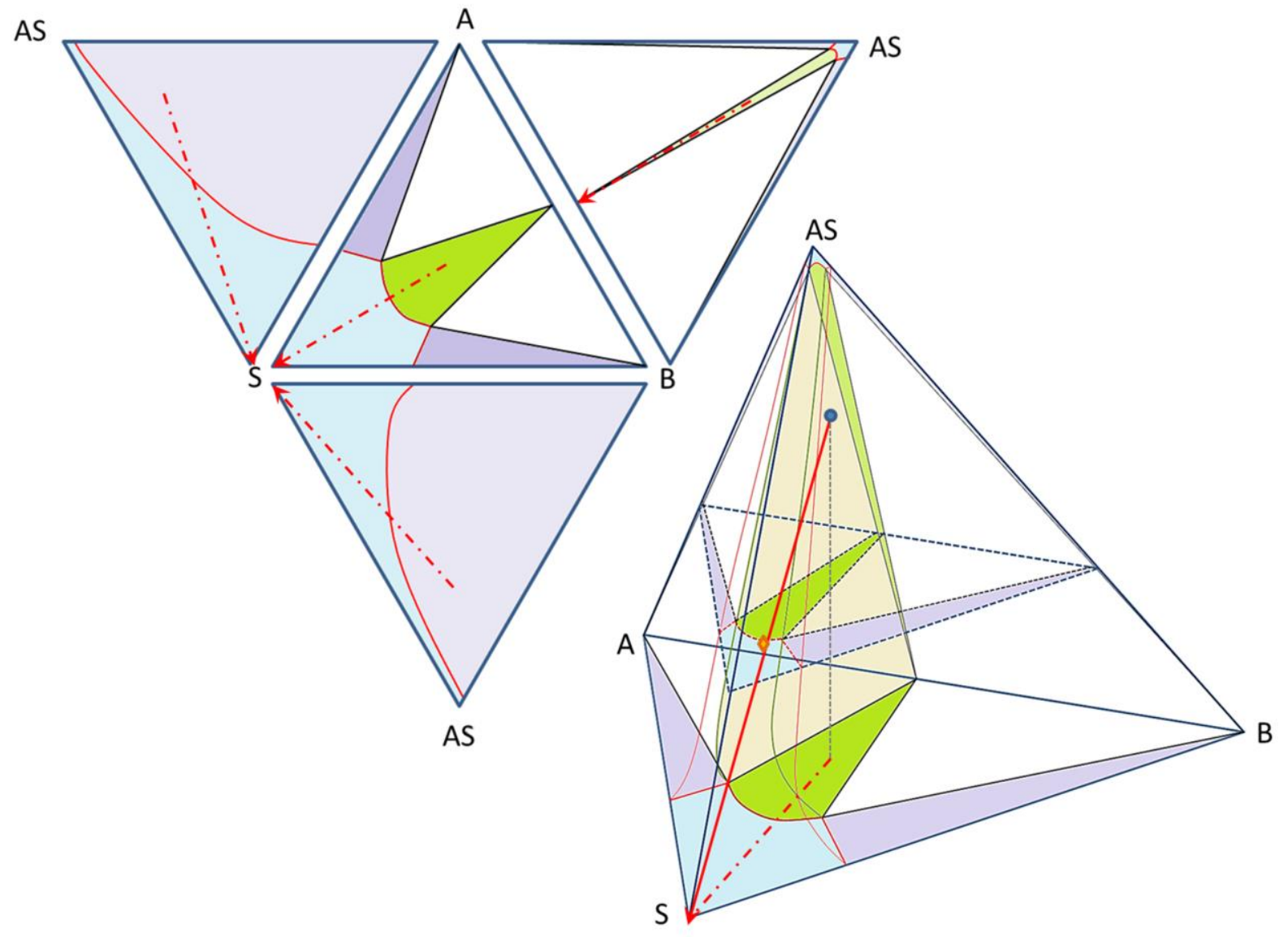

Figure 8. Quaternary phase diagram of a co-crystallization system in solvent (S) - anti-solvent (AS) mixtures with ternary phase diagrams colored at two different anti-solvent concentrations. Upper left: The phase diagram is unfolded into all ternary phase diagrams. Lower right: The quaternary phase diagram is depicted as a pyramid with the pure components (A, B, S and AS) at its corners. The green areas mark the co-crystal region, the lilac areas mark the regions where the pure components A or B crystallize, the blue areas mark a clear solution and the white areas mark the regions where the co-crystal is present together with either A or B. The red arrow depicts a possible solvent addition pathway, where pure solvent is added to a suspension in the co-crystal region. 


\section{Conclusion}

We found that the solvent addition method produces reliable solubility results for the organic compound p-hydroxybenzoic acid in the solvent acetic acid. The effect of changing the addition rate in solvent addition measurements follows a similar trend to the effect of changing the heating rate in the temperature variation method. Using low enough heating and addition rates, solubility data have been obtained that agree well with the more laborious suspension equilibrium method. The use of the SA method seems especially valuable in the case of solvent mixtures and other multi-component systems, e.g. with multiple solvents or solutes, in which the TV method cannot be applied easily. It was shown that the SA method works well for

determining a co-crystal phase diagram as well as a solvent mixture phase diagram for antisolvent crystallization. The SA measurements for the co-crystal system showed that the pure component solubilities are strongly influenced by the presence of the other component while that of the co-crystal is not. The SA method is therefore a suitable technique to investigate nonidealities in certain multi-component systems.

ASSOCIATED CONTENT

Supporting Information. N/A

AUTHOR INFORMATION

Corresponding Author

* Email: M.A.Reus@tudelft.nl

Present Addresses

N/A 


\section{Author Contributions}

The manuscript was written through contributions of all authors. All authors have given approval to the final version of the manuscript.

\section{Notes}

The authors declare no competing financial interest.

\section{ACKNOWLEDGMENT}

M. Hassan is acknowledged for his work on the INA-CBZ system, M. Stam and G. Scheuer are acknowledged for their experimental work on the DL-Asn system.

\section{ABBREVIATIONS}

EqC, Equilibrium Concentration; TV, Temperature Variation; SA, Solvent Addition; p-HBA, phydroxybenzoic acid; DL-Asn, DL-asparagine; CBZ, carbamazepine; INA, isonicotinamide.

\section{REFERENCES}

1. Mullin, J. W., Crystallization. 3 ed.; Butterworth-Heinemann Ltd.: Oxford, 1993.

2. $\quad$ Dufour, F.; Stichel, B.; Grayson, J. I., Org. Process Res. Dev. 2013, 17, 568-577.

3. Haas, C.; Drenth, J., J. Cryst. Growth 1999, 196, 388-394.

4. Chiarella, R. A.; Davey, R. J.; Peterson, M. L., Cryst. Growth Des. 2007, 7, 1223-1226.

5. $\quad$ Crocker, L. S.; Ge, Z.; Abrahim, A.; Hartman, R.; Xu, J., Org. Process Res. Dev. 2003, 7, 958-961.

6. Veesler, S.; Revalor, E.; Bottini, O.; Hoff, C., Org. Process Res. Dev. 2006, 10, 841-845.

7. Myerson, A., Handbook of Industrial Crystallization. Butterworth Heinemann2001.

8. Koutsoukos, P. G.; Kofina, A. N.; Kenellopoulou, D. G., Pure Appl. Chem. 2007, 79, 825-850.

9. Hermanto, M. W.; He, G.; Tjahjono, M.; Chow, P. S.; Tan, R. B. H.; Garland, M., Chem. Eng. Res. Des. 2011, 89, 2613-2619.

10. Vellema, J.; Hunfeld, N. G. M.; Van den Akker, H. E. A.; ter Horst, J. H., Eur. J. Pharm. Sci. 2011, 44, 621-626.

11. Srisanga, S.; ter Horst, J. H., Cryst. Growth Des. 2010, 10, 1808-1812.

12. Mullin, J. W.; Sipek, M., J. Chem. Eng. Data 1981, 26, 164-165.

13. Marchand, P.; Lefebvre, L.; Perez, G.; Counioux, J. J.; Coquerel, G., J. Therm. Anal. Calorim. 2002, 68, 37-47. 
14. Marchand, P.; Lefèbvre, L. c.; Querniard, F.; Cardinaël, P.; Perez, G.; Counioux, J.-J.; Coquerel, G., Tetrahedron: Asymmetry 2004, 15, 2455-2465.

15. Labarthe, E.; Bougrine, A. J.; Delalu, H.; Berthet, J.; Counioux, J. J., J. Therm. Anal. Calorim. 2009, 95, 135-139.

16. Berthet, J.; Tenu, R.; Counioux, J. J., Thermochim. Acta 1983, 68, 145-154.

17. Nordström, F. L.; Rasmuson, Å. C., J. Pharm. Sci. 2006, 95, 748-760.

18. ter Horst, J. H.; Deij, M. A.; Cains, P. W., Cryst. Growth Des. 2009, 9, 1531-1537.

19. Muller, F. L.; Fielding, M.; Black, S., Org. Process Res. Dev. 2009, 13, 1315-1321.

20. Good, D. J.; Rodríguez-Hornedo, N., Cryst. Growth Des. 2009, 9, 2252-2264.

21. Horst, J. H. t.; Cains, P. W., Cryst. Growth Des. 2008, 8, 2537-2542.

22. Hao, H.; Barrett, M.; Hu, Y.; Su, W.; Ferguson, S.; Wood, B.; Glennon, B., Org. Process Res. Dev. 2012, 16, 35-41.

23. Wang, G.; Ma, Y.; Wang, Y.; Hao, H.; Jiang, Y., Org. Process Res. Dev. 2015. 